\title{
ANÁLISE DE DISSERTAÇÕES E TESES EM EDUCAÇÃO E EDUCAÇÃO FÍSICA SOBRE ESTRATÉGIAS DE ENSINO E RECURSOS PEDAGÓGICOS PARA INCLUSÃO DO ALUNO COM DEFICIÊNCIA
}

Maria Luiza Salzani Fiorini, Universidade Estadual Paulista, UNESP, Marília, São Paulo - Brasil

Lígia Maria Presumido Braccialli, Universidade Estadual Paulista, UNESP, Marília, São Paulo - Brasil

Eduardo José Manzini, Universidade Estadual Paulista, UNESP, Marília, São Paulo Brasil

\section{RESUMO}

Objetivou-se realizar uma análise sistemática de Dissertações e Teses produzidas nos Programas de Pós-graduação em Educação e Educação Física e identificar as estratégias de ensino e os recursos pedagógicos que podem ser estabelecidos pelo professor de Educação Física na inclusão escolar do aluno com deficiência. A análise sistemática foi realizada por meio de cinco passos: 1) definir o objetivo da pesquisa; 2) buscar as Dissertações e Teses a partir da relação dos Programas de Pós-graduação em Educação e Educação Física avaliados pela Coordenação de Aperfeiçoamento de Pessoal de Nível Superior; 3) revisar e selecionar os estudos que atenderam os critérios de inclusão e exclusão; 4) analisar a qualidade metodológica dos estudos e, 5) apresentar os resultados. Os resultados foram discutidos a partir do ano/autor, Instituição, Programa de Pós-graduação, tipo, objetivo, desenho metodológico, participantes e resultados das Dissertações e Teses. Ao todo foram identificadas cinco Dissertações e três Teses com resultados referentes à estratégia de ensino e recurso pedagógico para a inclusão do aluno com deficiência nas aulas de Educação Física. As 129 estratégias de ensino identificadas foram para sete diferentes grupos de deficiência e, classificadas em quatro categorias (planejar ou preparar a aula, formar turmas, apresentar e ensinar uma atividade e, conduzir a aula). Os recursos pedagógicos identificados foram para as categorias deficiência intelectual e visual.

Palavras-Chave: Educação especial; Educação Física; Estratégia; Recurso.

\section{ANALYSIS OF DISSERTATIONS AND THESES ON EDUCATION AND PHYSICAL EDUCATION ABOUT EDUCATIONAL STRATEGIES AND PEDAGOGICAL RESOURCES FOR INCLUSION OF STUDENTS WITH DISABILITIES}

\footnotetext{
ABSTRACT

The research aimed to perform a systematic analysis of dissertations and theses produced in Education and Physical Education Postgraduate programs and identify teaching Conexões: revista da Faculdade de Educação Física da UNICAMP, Campinas, v. 13, n. 2, p. 98-116, abr./jun. 2015. ISSN: 1983-9030
} 
strategies and learning resources that can be established by a physical education teacher in the educational inclusion of student with disabilities. A systematic analysis was carried out in five steps: 1) define the purpose of the research; 2) look for the Dissertations and Theses from the the Graduate Program in Education and Physical Education reviewed by the Coordination of Improvement of Personnel with Higher Education; 3) review and select the studies that met the inclusion and exclusion criteria; 4) analyze the methodological quality of studies, and 5) present the results. The results were discussed from year/author, institution, Postgraduate Program, type, purpose, study design, participants, and results from the dissertations and theses. Altogether, it was identified five Dissertations and three Theses with results related to teaching strategy and pedagogical resource for the inclusion of students with disabilities in regular physical education classes. The 129 teaching strategies were identified for seven different disabilities groups and classified into four categories (planning or preparing your lesson, forming groups, presenting and teaching an activity and lead the class). The learning resources were identified for intellectual and visual disability categories.

Key-Words: Special education; Physical Education; Strategy; Resource.

\section{ANÁLISIS DE DISERTACIONES Y TESIS EN EDUCACIÓN Y EDUCACIÓN FÍSICA SOBRE ESTRATEGIAS DE ENSEÑANZA Y RECURSOS PEDAGÓGICOS PARA INCLUSIÓN DEL ALUMNO CON DISCAPACIDAD}

\section{RESUMEN}

La Investigación tuvo como objetivo realizar un análisis sistemático de Disertaciones y Tesis producidas en los Programas de Posgrado en Educación y Educación Física e identificar las estrategias de enseñanza y los recursos pedagógicos que pueden ser establecidos por el profesor de Educación Física en la inclusión educacional del alumno con discapacidad. El análisis sistemático fue realizado por medio de cinco pasos: 1) definir el objetivo de la investigación; 2) buscar las Disertaciones y Tesis a partir de la relación de los Programas de Posgrado en Educación y Educación Física evaluados por la Coordinación de Perfeccionamiento de Personal de Nivel Superior; 3) repasar y seleccionar los estudios que atendieron a los criterios de inclusión y exclusión; 4) analizar la cualidad metodológica de los estudios y, 5) presentar los resultados. Los resultados fueron discutidos a partir del año/autor, Institución, Programa de Posgrado, tipo, objetivo, diseño metodológico, participantes y resultados de las Disertaciones y Tesis. En total fueron identificadas cinco Disertaciones y tres Tesis con resultados referentes a la estrategia de enseñanza y recurso pedagógico para la inclusión del alumno con discapacidad en las clases de Educación Física. Las 129 estrategias de enseñanza identificadas fueron para siete diferentes grupos de discapacidad y, clasificadas en cuatro categorías (planear o preparar la clase, formar grupos, presentar y enseñar una actividad y, conducir la clase). Los recursos pedagógicos identificados fueron para las categorías discapacidad intelectual y visual. 
Palabras-Clave: Educación especial; Educación Física; Estrategia; Recurso.

Conexões: revista da Faculdade de Educação Física da UNICAMP, Campinas, v. 13, n. 2, p. 98-116, abr./jun. 2015. ISSN: 1983-9030 


\section{INTRODUÇÃO}

As aulas de Educação Física, por meio dos eixos de conteúdos que representam a área, promovem atividades como esportes, danças, ginásticas, jogos e lutas. ${ }^{1-2}$ Nessas aulas, percebe-se que o professor necessita estabelecer estratégias de ensino e selecionar recursos pedagógicos adequados e/ou adaptados para que seu alunado desempenhe, adequadamente, as várias atividades propostas. ${ }^{3-5}$ Esse transcurso habitual das aulas parece não ser tão tranquilo quando o professor defronta-se com alunos com deficiência matriculados no Ensino Regular, uma vez que necessita de um conhecimento mais aprofundado sobre como lidar, ensinar e propor atividades para essa população. ${ }^{6}$ A questão para o professor é como vislumbrar, ao mesmo tempo, as capacidades, as limitações, as necessidades e os cuidados básicos de atendimento e segurança a alunos com deficiência matriculados de forma a tornar exequível a sua participação. ${ }^{4}$

Cabe aqui definir os termos estratégia de ensino e recurso pedagógico. Por estratégia de ensino adota-se a definição de Manzini, ${ }^{7}$ elaborada para a área educacional, em que a estratégia é uma ação do professor, que muitas vezes, faz uso de um recurso pedagógico para alcançar um objetivo de ensino ou de avaliação, e que pode ser identificada pelos verbos de ações das orações no plano de ensino do professor. ${ }^{7}$ A estratégia é flexível, passível de ser modificada e direciona a ação do professor e do aluno. ${ }^{7}$ Dessa forma, a estratégia pode ser entendida como uma ferramenta que exige a compreensão dos requisitos necessários para preparar e aplicar tarefas motoras frente as necessidades e capacidades da clientela em questão. ${ }^{4} \mathrm{O}$ exercício de planejar as estratégias a serem estabelecidas pelo professor, em uma determinada aula, é fundamental para que sejam minimizadas as tentativas e erros ou os improvisos durante as aulas. ${ }^{8}$

Por sua vez, o recurso pedagógico é um objeto que possui três componentes: 1) ser concreto; 2) manipulável; 3) ter uma finalidade pedagógica (de ensino). ${ }^{9-10}$ Com base nessa definição, entende-se que os recursos pedagógicos são materiais e/ou implementos utilizados para: execução, ensino, treinamento, suporte e aprendizado de tarefas motoras frente as necessidades e capacidades da clientela em questão. ${ }^{4}$

Conexões: revista da Faculdade de Educação Física da UNICAMP, Campinas, v. 13, n. 2, p. 98-116, abr./jun. 2015. ISSN: 1983-9030 
As estratégias de ensino e os recursos pedagógicos para alunos com deficiência fazem parte da denominada Tecnologia Assistiva, cuja definição foi elaborada pelo Comitê Nacional de Ajudas Técnicas com vistas à realidade brasileira:

Tecnologia Assistiva é uma área do conhecimento, de característica interdisciplinar, que engloba produtos, recursos, metodologias, estratégias, práticas e serviços que objetivam promover a funcionalidade, relacionada à atividade e participação, de pessoas com deficiência, incapacidades ou mobilidade reduzida, visando sua autonomia, independência, qualidade de vida e inclusão social. ${ }^{11: 3}$

Especificamente sobre estratégias de ensino para a inclusão escolar de alunos com deficiência nas aulas regulares de Educação Física, as questões de ordem são: o que fazer? Como fazer? ${ }^{12-14}$ A busca por respostas sobre "como conduzir uma aula de Educação Física para turmas regulares em que há alunos com deficiência matriculados" encontra um fator influenciador: a formação. ${ }^{15}$ Os resultados da pesquisa de Fiorini ${ }^{16}$ indicaram que os professores de Educação Física, participantes, reconheciam a necessidade de estratégias de ensino adequadas em turmas que havia alunos com e sem deficiência, mas os professores não souberam dizer quais poderiam ser as estratégias.

Essa dúvida que permeia a questão das estratégias de ensino não é algo exclusivo da Educação Física, mas da Educação como um todo. Para Manzini, ${ }^{7}$ a experiência na formação de professores demonstrou a existência de grande dificuldade ao tentar definir o que é uma estratégia e o que é um recurso. Reganhan e Manzini, ${ }^{17}$ ao observarem os relatos de professores do Ensino Regular, identificaram a dificuldade deles perceberem e descreverem as estratégias de ensino. Os autores salientaram que este parece ser um problema de formação conceitual, necessário para o ensino de alunos com ou sem deficiência. O conhecimento, por parte dos professores, sobre as estratégias de ensino e suas possíveis adaptações, utilizados no decorrer da prática docente, estão ligados diretamente aos conhecimentos obtidos pelo processo de formação do professor, inicial e continuada. $^{18}$

Diante do exposto, este estudo apresenta como problema de pesquisa: quais estratégias de ensino e recursos pedagógicos são indicados na literatura nacional para que o professor de Educação Física possa utilizar na inclusão escolar do aluno com deficiência? 


\section{OBJETIVO}

Realizar uma análise sistemática de Dissertações e Teses produzidas nos Programas de Pós-Graduação em Educação e Educação Física e identificar as estratégias de ensino e os recursos pedagógicos que podem ser estabelecidos pelo professor de Educação Física na inclusão escolar do aluno com deficiência.

\section{MÉTODO}

Optou-se por realizar uma revisão sistemática definida como:

[...] uma forma de pesquisa que utiliza como fonte de dados a literatura sobre determinado tema. Esse tipo de investigação disponibiliza um resumo das evidências relacionadas a uma estratégia de intervenção específica, mediante a aplicação de métodos explícitos e sistematizados de busca, apreciação crítica e síntese da informação selecionada. ${ }^{19: 84}$

Os cinco passos descritos por Sampaio e Mancini ${ }^{19}$ como necessários para o processo de elaboração de um estudo de revisão sistemática foram seguidos, sendo eles: 1) definindo a pergunta; 2) buscando a evidência; 3) revisando e selecionando os estudos; 4) analisando a qualidade metodológica do estudo, e 5) apresentando os resultados.

O primeiro passo foi definir a pergunta ou objetivo da pesquisa, que foi identificar estratégias de ensino e recursos pedagógicos que podem ser estabelecidos e selecionados pelo professor de Educação Física na inclusão escolar do aluno com deficiência no Sistema Regular de Ensino.

No segundo passo, definiu-se a estratégia de busca, a qual compreendeu a partir da relação dos Programas de Pós-Graduação em Educação e Educação Física avaliados pela Coordenação de Aperfeiçoamento de Pessoal de Nível Superior (CAPES/MEC) no triênio 2007-2009, ${ }^{20-21}$ acessar os sites de cada um dos 92 Programas de Pós-graduação em Educação (PPGE) e 37 Programas de Pós-graduação em Educação Física (PPGEF) e verificar nos Programas de Pós-graduação em Educação, os que possuíam a área/linha Educação Especial ou afim, e nos Programas de Pós-graduação em Educação Física, os que possuíam a área/linha Educação Física Adaptada ou afim. Os critérios de inclusão Conexões: revista da Faculdade de Educação Física da UNICAMP, Campinas, v. 13, n. 2, p. 98-116, abr./jun. 2015. ISSN: 1983-9030 
foram: 1) Dissertações e Teses produzidas nos PPGE, na área/linha Educação Especial ou afim, com o tema inclusão do aluno com deficiência nas aulas de Educação Física no Sistema Regular de Ensino; 2) Dissertações e Teses produzidas nos PPGEF, na área/linha Educação Física Adaptada ou afim, com o tema inclusão do aluno com deficiência nas aulas de Educação Física no Sistema Regular de Ensino e, 3) Dissertações e Teses disponíveis no site do Programa ou na Biblioteca Digital da instituição de ensino até o período de realização da pesquisa (2011). Foram excluídas as Dissertações e Teses em que a abordagem metodológica foi a de revisão bibliográfica.

O terceiro passo contemplou a seleção e revisão dos estudos. A partir da busca realizada e dos critérios expostos foram identificados 11 Programas de Pós-graduação em Educação com a área/linha Educação Especial ou afim (UERG, USP, UFES, UFRGS, UFRN, Unesp/Marília, UFBA, UFSM, UEL, UFGD, UNISC) e um Programa de Pós-graduação em Educação Especial (UFSCar). Quanto a Educação Física, foi identificado apenas um Programa de Pós-graduação com a área/linha Educação Física Adaptada ou afim (UNICAMP). Ao todo foram identificadas sete Dissertações e uma Tese nos PPGE, uma Tese no Programa de Pós-graduação em Educação Especial e, sete Dissertações e duas Teses no PPGE sobre inclusão do aluno com deficiência nas aulas de Educação Física no Ensino Regular.

No quarto passo foi realizada a análise da qualidade metodológica dos estudos identificados, por meio da leitura do título, resumo, objetivo e resultados. Buscou-se responder cinco questões: 1) A Dissertação/Tese apresenta dados referentes à estratégia de ensino e recursos pedagógicos? 2) Qual é a estratégia de ensino? 3) A estratégia é específica para algum tipo de deficiência? 4) Qual é o recurso pedagógico? 5) O recurso é específico para algum tipo de deficiência? As palavras aceitas foram adaptação, estratégia, verbos que indicassem ação do professor, recurso e material.

No quinto passo os resultados foram organizados.

\section{RESULTADOS E DISCUSSÃO}

Conexões: revista da Faculdade de Educação Física da UNICAMP, Campinas, v. 13, n. 2, p. 98-116, abr./jun. 2015. ISSN: 1983-9030 
Dentre as 14 Dissertações e quatro Teses analisadas, cinco Dissertações e três Teses possuíam dados referentes às estratégias de ensino e recursos pedagógicos para inclusão escolar do aluno com deficiência nas aulas de Educação Física. A discussão dos resultados pautar-se-á nessas oito produções.

Os resultados serão apresentados a partir de cinco características das Dissertações e Teses analisadas que são: ano/autor, instituição, Programa de Pós-graduação, tipo, objetivos, desenho metodológico, participantes e resultados (estratégias e recursos pedagógico).

\section{Ano/autor}

Os autores das cinco Dissertações e três Teses serão apresentados por ordem cronológica de defesa: Florence (2002), Moraes (2004), Cruz (2005), Souza (2008), Bezerra (2010), Feijó (2011), Fiorini (2011) e Valverde (2011).

As Dissertações e Teses com resultados referentes à estratégia de ensino e recurso pedagógico para a inclusão escolar de alunos com deficiência nas aulas de Educação Física começaram a ser descritos a partir de 2002 e tiveram uma crescente a partir de 2010.

A hipótese é que as Dissertações e Teses acompanharam, e ainda acompanham, as etapas do processo de inclusão de alunos com deficiência e os seus resultados refletem os retrocessos, estagnações e avanços, isso é, até os anos 90 havia uma luta pelos direitos desses alunos, a preocupação estava em matriculá-los nas escolas regulares. Hoje, a inclusão escolar é uma realidade, e por isso, a preocupação não é mais em garantir que os alunos com deficiência frequentem às escolas do Ensino Regular, mas buscam-se formas e meios de criar condições que favoreçam a inclusão escolar de alunos com deficiência.

\section{Instituição}

As Dissertações e Teses que abordaram o tema inclusão e que tiverem resultados relativos à estratégia de ensino e recurso pedagógico eram vinculadas á quatro Universidades (UFSCar, Unesp/Marília, UNICAMP e UEL). 
Muito provavelmente esse resultado se deva ao perfil traçado por essas Universidades frente às questões da Educação Especial, por exemplo, a UFSCar e a Unesp/Marília que se destacam no cenário em nível estadual e nacional e, a UNICAMP como referência quando na Educação Física Adaptada.

\section{Programa de Pós-Graduação}

Dentre os 92 PPGE e 37 PPGEF, as referidas Dissertações e Teses foram defendidas em dois PPGE (UEL e Unesp/Marília), um Programa de Pós-graduação em Educação Especial (UFSCar) e um PPGEF (UNICAMP).

Tal número representou 0,97\% do total de Dissertações do PPGE Unesp/Marília e 1,81\% das Dissertações disponíveis no site do Programa da UEL. As Teses representam 0,675\% do total de Teses do Programa do PPGE Unesp/Marília e 1,67\% das Teses disponíveis no Programa de Pós-Graduação em Educação Especial, da UFSCar. Com relação ao PPGEF da UNICAMP, as Dissertações e Teses, juntas, representam 0,30\% do número total de Dissertações e Teses do Programa.

Cabe aqui mencionar que há, no Brasil, apenas um Programa de Pós-Graduação em Educação Física com área ou linha de pesquisa em Educação Física Adaptada, o da UNICAMP. Em contrapartida, há 11 Programas de Pós-graduação em Educação com a área/linha Educação Especial ou afim e um Programa de Pós- graduação em Educação Especial.

\section{Tipo}

O tipo de produção se refere ao fato de ser Dissertação ou Tese. No quesito estratégias de ensino e recursos pedagógicos para a inclusão nas aulas de Educação Física, as Dissertações foram maioria, isso porque, do total de oito publicações foram cinco Dissertações ${ }^{3,16,22-24}$ e três Teses. ${ }^{25-27}$

\section{Objetivos}

Conexões: revista da Faculdade de Educação Física da UNICAMP, Campinas, v. 13, n. 2, p. 98-116, abr./jun. 2015. ISSN: 1983-9030 
A partir dos objetivos das Dissertações e Teses analisadas foi possível estabelecer três categorias.

A primeira categoria compreendeu as Dissertações e Teses com objetivos que especificaram claramente o termo estratégia como a Tese de Bezerra ${ }^{27}$ da Unesp/Marília e a Dissertação de Valverde ${ }^{24}$ da UEL.

$\mathrm{Na}$ segunda categoria foram arroladas as Teses que não tiveram o termo estratégia descrito no objetivo, porém, delinearam objetivos que remeteram à ideia de estratégias de ensino diante da inclusão de alunos com deficiência, como foi o caso da Tese de Cruz, ${ }^{25}$ da UNICAMP e a Tese de Souza $^{26}$ da UFSCar. Por exemplo, acompanhar como os professores lidam com a inclusão e apontar como um programa de formação continuada pode ampliar o instrumental do professor de Educação Física.

E, a terceira categoria contemplou as outras quatro Dissertações, as quais tiveram objetivos de pesquisa mais amplos, focados na temática inclusão e como consequência, chegaram a resultados sobre estratégias.

Nenhuma Dissertação ou Tese teve como objetivo específico identificar ou descrever recursos pedagógicos para a inclusão escolar de alunos com deficiência nas aulas regulares de Educação Física.

\section{Desenho metodológico}

A partir das informações advindas das Dissertações e Teses analisadas foi possível identificar os tipos de pesquisa, os procedimentos de coleta e os instrumentos utilizados.

Com relação ao tipo de pesquisa foram mencionados cinco diferentes tipos: pesquisa-ação, estudo de caso, estudo descritivo, pesquisa etnográfica e pesquisa de intervenção.

Quanto aos procedimentos de coleta de dados: 1) seis produções utilizaram entrevista semiestruturada; 2) quatro realizaram observação; 3) três utilizaram filmagem; 4) uma produção usou grupo focal e, 5) uma produção utilizou diário de campo.

Conexões: revista da Faculdade de Educação Física da UNICAMP, Campinas, v. 13, n. 2, p. 98-116, abr./jun. 2015. ISSN: 1983-9030 
Do total de cinco Dissertações e três Teses, três dessas publicações utilizaram apenas um procedimento de coleta de dados, uma fez uso de dois procedimentos e quatro usaram mais de três procedimentos para coletar os dados da pesquisa.

Como instrumentos de coleta de dados foram utilizados roteiro de entrevista em seis Dissertações/Teses, questionário de elaboração própria em uma Dissertação/Tese e, uma escala pronta, de outra autoria, em uma Dissertação/Tese.

\section{Participantes}

Esse tópico será discutido a partir de três variáveis: 1) o tipo; 2) o número e a relação com o procedimento de coleta de dados e, 3 ) as redes de ensino.

Quanto ao tipo foi possível identificar que os autores consideraram como participantes os próprios professores de Educação Física, os alunos com deficiência e os alunos sem deficiência.

Em relação ao número de participantes a Dissertação/Tese com menor número de participantes foi de seis professores de Educação Física e a Dissertação/Tese com o maior número de participantes foi de 146, somados professor e alunos com e sem deficiência.

O número de participantes foi variado, também, em função do procedimento de coleta de dados realizado, por exemplo, nas Dissertações e Teses com os menores números de participantes: realização de entrevista semiestruturada com seis participantes; pesquisa de intervenção com realização de treinamento de tutores envolvendo sete participantes (um professor, um aluno com deficiência e cinco aluno sem deficiência); pesquisa de intervenção com formação continuada de professores de Educação Física por meio de grupo focal, observação e filmagem com 16 professores participantes e, pesquisa etnográfica com 29 participantes, somados professor de Educação Física e alunos com e sem deficiência. 
Nas Dissertações e Teses com número mais elevado de participantes houve a realização de filmagem com 146 participantes, sendo eles, professor e alunos com e sem deficiência e, a aplicação de questionário com 65 professores de Educação Física.

\section{Resultados (estratégias e recursos pedagógico)}

A partir da análise das Dissertações e Teses foram identificadas estratégias de ensino para sete diferentes grupos: 1) independente do tipo; 2) deficiência física; 3) condutas típicas; 4) deficiência intelectual; 5) autismo; 6) deficiência auditiva e, 7) deficiência visual. Nota-se que houve estratégias tanto para alunos com algum tipo de deficiência quanto para alunos com Transtorno Global de Desenvolvimento (TGD) e, não foram identificadas estratégias para alunos com altas habilidades/superdotação.

Algumas Dissertações/Teses continham estratégias específicas para uma categoria, como também, estratégias mais abrangentes e que poderiam ser estabelecidas, pelo professor de Educação Física, independentemente da categoria anteriormente mencionada.

No total foram identificadas 129 estratégias. Para a categoria independente do tipo foram 17; para deficiência física 36; para condutas típicas foram identificadas seis estratégias; para deficiência intelectual 48; para autismo cinco; para deficiência auditiva sete estratégias e, para deficiência visual foram 10 estratégias.

As 129 estratégias foram classificadas em quatro categorias:

1) Para planejar ou preparar a aula: conhecer a deficiência do aluno e as dificuldades em razão da deficiência; planejar a aula para que o aluno com deficiência se adapte a ela; planejar uma atividade que todos possam participar ao invés de elaborar algo isolado para o aluno com deficiência; planejar atividades diferenciadas para aluno com deficiência ou então jogos de tabuleiro; adaptar as atividades visando a segurança do aluno; programar a aula de modo a conseguir um equilíbrio entre a resposta do grupo e de cada aluno individualmente; trabalhar com atividades diferenciadas; preparar o ambiente de modo a ser seguro; adaptar os recursos; solicitar sugestões dos alunos; planejar uma aula mais movimentada e agitada; tratar os alunos com igualdade; escolher o conteúdo da aula (jogo,

Conexões: revista da Faculdade de Educação Física da UNICAMP, Campinas, v. 13, n. 2, p. 98-116, abr./jun. 2015. ISSN: 1983-9030 
esporte, competição, cooperação); planejar as atividades "1 e 2" caso a primeira não funcione;

2) Para formar turmas: enumerar as crianças; evitar colunas ou fileiras; dispor em fileiras e não em colunas; montar pequenos grupos de no máximo três alunos cada; misturar meninos e meninas; trabalhar em círculo; integrar alunos de faixa etária quase igual;

3) Para apresentar e ensinar uma atividade: passar para o aluno o conhecimento dos conteúdos (o que estão fazendo e porque); utilizar técnicas de ajuda (dica verbal, assistência física I - aluno toca no professor ou colega para perceber o movimento; assistência física II - professor ou colega toca no aluno e realiza o movimento); oferecer feedback; usar linguagem acessível; oferecer uma instrução geral a todos os alunos sobre a atividade; adaptar as regras; usar materiais concretos, figuras, imagens ou qualquer recurso de natureza manipulável; demonstrar o movimento e o aluno observa; permanecer de frente para o aluno, verbalizar o exercício e o aluno faz a leitura labial; em aulas teóricas, escrever o conteúdo na lousa; ter um Intérprete de Libras e, "ditar" o conteúdo ao invés de escrever na lousa;

4) Para conduzir a aula: ter um auxiliar; colocar o aluno na cadeira de rodas para poupar o professor e libera-lo para atender outros alunos; solicitar ajuda de um colega da sala (Tutor); manter os alunos o tempo todo com material; dominar a sala de aula; conduzir a aula de modo a respeitar uma sequência pedagógica (progressão); motivar continuamente o aluno; tornar o aluno líder e ajudante.

Dentre as estratégias identificadas quase que a totalidade referiu-se às aulas práticas de Educação Física, porém, também foram mencionadas estratégias para aulas teóricas de Educação Física, especificamente diante da matrícula de alunos com deficiência auditiva e visual. Aula teórica de Educação Física é uma realidade nas escolas Estaduais do ciclo II e Ensino Médio, do Estado de São Paulo, a partir da Proposta Curricular do Estado de São Paulo. ${ }^{2,28}$ 
Cabe aqui mencionar uma questão relativa à estratégia "para formar turmas", isso porque, não houve um consenso se a estratégia mais adequada era dispor os alunos em colunas ou fileiras, uma vez que foi indicado evitar ambos (trabalho em fileiras e não em colunas; evitar colunas ou fileiras).

A escolha por dispor os alunos em coluna, fileira ou círculo remete à ideia de segurança dos alunos com deficiência que, em qualquer dessas disposições, terão colegas como referência, tanto para auxiliares quanto para modelo.

Em relação aos recursos pedagógicos foram identificados para alunos com deficiência visual, por exemplo, apito, bolas coloridas e guizo e, para a deficiência intelectual, por exemplo, materiais concretos, figuras, imagens ou qualquer recurso de natureza manipulável.

\section{CONCLUSÃO}

Conclui-se, a partir da revisão sistemática que apenas cinco Dissertações e três Teses continham resultados sobre estratégias de ensino e recursos pedagógicos para a inclusão escolar de alunos com deficiência nas aulas de Educação Física.

As referidas Dissertações e Teses foram publicadas no período de 2002 a 2011 e estavam vinculadas a quatro Universidades (UFSCar, Unesp/Marília, UNICAMP e UEL).

Dentre os 92 PPGE e 37 PPGEF, as Dissertações e Teses foram defendidas em dois PPGE (UEL e Unesp/Marília), um Programa de Pós-graduação em Educação Especial (UFSCar) e um PPGEF (UNICAMP).

Quanto aos objetivos de pesquisa, houve os que especificaram claramente o termo estratégia; os que não tinham o termo estratégia descrito, porém, remetia á ideia de estratégia de ensino diante da inclusão; os mais amplos e focados na temática da inclusão e como consequência obtiveram resultados sobre estratégias e, nenhum objetivo específico para recursos.

Conexões: revista da Faculdade de Educação Física da UNICAMP, Campinas, v. 13, n. 2, p. 98-116, abr./jun. 2015. ISSN: 1983-9030 
Com relação ao desenho metodológico foram realizados pesquisa-ação, estudo de caso, estudo descritivo, pesquisa etnográfica e pesquisa de intervenção; os procedimentos de coleta de dados envolveram entrevista semiestruturada, observação, filmagem, grupo focal e diário de campo e, os instrumentos foram roteiro de entrevista, questionário e escala.

Considerou-se como participantes os professores de Educação Física e os alunos com e sem deficiência. O número de participantes variou de seis a 146.

Ao todo foram identificadas 129 estratégias para sete diferentes grupos: 1) independente do tipo; 2) deficiência física; 3) condutas típicas; 4) deficiência intelectual; 5) autismo; 6) deficiência auditiva e, 7) deficiência visual. Tais estratégias para foram indicadas para planejar ou preparar a aula, formar turmas, apresentar e ensinar uma atividade e, conduzir a aula em aula teórica e prática.

Os recursos pedagógicos identificados foram específicos para ser em utilizados pelos professores de Educação Física, nas aulas regulares, quando há alunos com deficiência intelectual e alunos com deficiência visual.

A temática da inclusão do aluno com deficiência nas aulas de Educação Física tem sido alvo de Dissertações e Teses nos Programas de Pós-Graduação em Educação, em Educação Especial e em Educação Física, porém, ainda é pequena a produção que tenha como foco estratégias de ensino e recursos pedagógicos.

\section{REFERÊNCIAS}

${ }^{1}$ BRASIL. Ministério da Educação e Cultura. Secretaria de Educação Fundamental. Parâmetros Curriculares Nacionais. Brasília, 1998.

\section{${ }^{2}$ SÃO PAUlO (Estado). SECRETARIA DO ESTADO DA EDUCAÇÃO. Proposta} curricular do estado de São Paulo: Educação Física. São Paulo, 2008. 
${ }^{3}$ FLORENCE, R. B. P. A educação física na rede pública do município de São João da Boa Vista-SP e o portador de necessidades especiais: do direito ao alcance. 2002. $109 \mathrm{f}$. Dissertação (Mestrado em Educação Física) - Universidade Estadual de Campinas, Campinas, 2002.

${ }^{4}$ SEABRA JÚNIOR, M. O.; MANZINI, E. J. Recursos e estratégias para o ensino do aluno com deficiência visual na atividade física adaptada. Marília: ABPEE, 2008.

${ }^{5}$ SILVA, A. M.; MENDES, E. G. Família de crianças com deficiência e profissionais: componentes da parceria colaborativa na escola. Revista Brasileira de Educação Especial, Marília, v.14, n. 2, p. 217-234, 2008.

${ }^{6}$ FIORINI, M. L. S.; MANZINI, E. J. Dificuldades dos professores de Educação Física diante da inclusão educacional de alunos com deficiência. In: CONGRESSO BRASILEIRO DE EDUCAÇÃO ESPECIAL, 5., 2012, São Carlos. Anais... São Carlos: Cubo, 2012.p. $8844-8858$

${ }^{7}$ MANZINI, E. J. Recurso pedagógico adaptado e estratégias para o ensino de alunos com deficiência física In: MANZINI, E. J.; FUJISAWA, D. S. (Org.). Jogos e recursos para comunicação e ensino na educação especial. Marília: ABPEE, 2010. p. 117-138.

${ }^{8}$ SILVA, M. O. Protocolo para prescrição ou adaptação de recursos pedagógicos para alunos com paralisia cerebral. 2010. 195f. Dissertação (Mestrado) - Faculdade de Filosofia e Ciências, Universidade Estadual Paulista, Marília, 2010.

${ }^{9}$ MANZINI, E. J. Recursos pedagógicos para o ensino de alunos com paralisia cerebral. Revista Mensagem da Apae, v. 36, n. 84, p.17-21, 1999.

${ }^{10}$ MANZINI, E. J.; DELIBERATO, D. Portal de ajudas técnicas: equipamento e material pedagógico para educação - recursos adaptados II. Brasília: ABPEE/MEC/SEESP, 2007. 
${ }^{11}$ BRASIL. Presidência da República. Secretaria Especial dos Direitos Humanos. Ata da reunião VII, de dezembro de 2007 do Comitê de Ajudas Técnicas. Disponível em: <http://www.mj.gov.br/corde/arquivos/doc/Ata\%20III\%2019\%20e\%2020\%20abril2007.d oc>. Acesso em: 20 jul. 2011.

${ }^{12}$ FILUS, J. F.; MARTINS JUNIOR, J. Inclusão de pessoas com deficiência na escola: a opinião dos professores de educação física. Acta Scientiarum. Human and Social Sciences, Maringá, v. 26, n. 1, p. 103-108, 2004.

${ }^{13}$ BRITO, R. F. A.; LIMA, J. F. Desafios encontrados pelos professores de educação física no trabalho com alunos com deficiência. Corpo, Movimento e Saúde, Salvador, v. 2, n. 1, p.1-12, 2012.

${ }^{14}$ CHICON, J. F.; CRUZ, G. C. Educação física inclusiva: formação e prática pedagíca. In: VICTOR, S. L.; DRAGO, R.; PANTALEÃO, E. (Org.). Educação especial: indícios, registros e práticas de inclusão. São Carlos: Pedro \& João, 2013.

${ }^{15}$ FIORINI, M. L. S.; MANZINI, E. J. Formação do professor educação física para inclusão de alunos com deficiência. Poiesis Pedagógica, Catalão, v.12, n.1, p. 94-109, 2014.

${ }^{16}$ FIORINI, M. L. S. Concepção do professor de educação física sobre a inclusão do aluno com deficiência. 2011. 143 f. Dissertação (Mestrado em Educação) Faculdade de Filosofia e Ciências, Universidade Estadual Paulista, Marília, 2011.

${ }^{17}$ REGANHAN, W. G.; MANZINI, E. J. Percepção de professores do ensino regular sobre recursos e estratégias para o ensino de alunos com deficiência. Revista Educação Especial, Santa Maria, v. 22, n. 34, p. 127-138, 2009.

${ }^{18}$ CAPARROZ, F. E.; BRACHT, V. O tempo e o lugar de uma didática da educação física. Revista Brasileira de Ciências do Esporte, Campinas, v. 28, n. 2, p. 21-47, 2007.

Conexões: revista da Faculdade de Educação Física da UNICAMP, Campinas, v. 13, n. 2, p. 98-116, abr./jun. 2015. ISSN: 1983-9030 
SAMPAIO, R. F.; MANCINI, M. C. Estudos de revisão sistemática: um guia para síntese criteriosa da evidência científica. Revista Brasileira de Fisioterapia, São Carlos, v.11, n.1, p. 83-89, 2007.

${ }^{20}$ COORDENAÇÃO DE APERFEIÇOAMENTO DE PESSOAL DE NÍVEL SUPERIOR (CAPES). Produção bibliográfica distribuída segundo a estratificação Qualis, Teses e Dissertações defendidas e número de Docentes permanentes, triênio 2007-09, e Nota final da Avaliação 2010: Área de avaliação: Educação. 2010a. Disponível em:< http://www.capes.gov.br/component/content/article/44-avaliacao/4355planilhascomparativas-da-avaliacao-trienal-2010>. Acesso em: 21 set. 2011.

${ }^{21}$ COORDENAÇÃO DE APERFEIÇOAMENTO DE PESSOAL DE NÍVEL SUPERIOR (CAPES). Produção bibliográfica distribuída segundo a estratificação Qualis, Teses e Dissertações defendidas e número de Docentes permanentes, triênio 2007-09, e Nota final da Avaliação 2010 - Área de avaliação: Educação Física. 2010b. Disponível em:< http://www.capes.gov.br/images/stories/download/avaliacaotrienal/planilhascomparat ivastrienal2010/Educacao_Fisica.xls>. Acesso em: 21 set. 2011.

${ }^{22}$ MORAES, A. C. F. Contribuições da disciplina de educação física para a educação inclusiva de alunos com deficiência: o ponto de vista dos professores. 2004. $98 \mathrm{f}$. Dissertação (Mestrado em Educação) - Faculdade de Filosofia e Ciências, Universidade Estadual Paulista, Marília, 2004.

${ }^{23}$ FEIJÓ, G. O. Classe especial: os desafios dos professores de Educação Física para a inclusão de alunos com condutas típicas. 2011. 98 f. Dissertação (Mestrado em Educação) - Centro de Educação, Comunicação e Artes, Universidade Estadual de Londrina, Londrina, 2011.

${ }^{24}$ VALVERDE, G. S. A docência em Educação Física: atuação em meio inclusivo de professores ex-atleta e não atletas. 2011. 95 f. Dissertação (Mestrado em Educação) Universidade Estadual de Londrina, Londrina, 2011

Conexões: revista da Faculdade de Educação Física da UNICAMP, Campinas, v. 13, n. 2, p. 98-116, abr./jun. 2015. ISSN: 1983-9030 
${ }^{25}$ CRUZ, G. C. Formação continuada de professores de educação física em ambiente escolar inclusivo. 2005. 254 f. Tese (Doutorado) - Faculdade de Educação Física, Universidade Estadual de Campinas, Campinas, 2005.

${ }^{26}$ SOUZA, J. V. Tutoria: estratégias de ensino para inclusão de alunos com deficiência em aulas de educação física. 2008. 136 f. Tese (Doutorado em Educação Especial) Universidade Federal de São Carlos, São Carlos, 2008.

${ }^{27}$ BEZERRA, A. F. S. Estratégias para o ensino inclusivo de alunos com deficiência nas aulas de Educação Física. 2010. 108 f. Tese (Doutorado em Educação) - Faculdade de Filosofia e Ciências, Universidade Estadual Paulista, Marília, 2010.

${ }^{28}$ SÃO PAULO (Estado). Secretaria do Estado da Educação. Caderno do professor: educação física, ensino fundamental - $8^{\mathbf{a}}$ série, $3^{\circ}$ bimestre. São Paulo, 2008.

Recebido em: 04 set. 2014

Aceito em: 30 mar. 2015

Contato: mazinhasf@yahoo.com.br

Conexões: revista da Faculdade de Educação Física da UNICAMP, Campinas, v. 13, n. 2, p. 98-116, abr./jun. 2015.

ISSN: 1983-9030 